\begin{tabular}{|c|c|}
\hline Title & $\begin{array}{l}\text { Chaotic itinerancy as a mechanism of irregular changes between synchronization and desynchronization in a neural } \\
\text { network }\end{array}$ \\
\hline Author(s) & Tsuda, Ichiro; Fujii, Hiroshi; Tadokoro, Satoru; Y asuoka, Takui; Y amaguti, Y utaka \\
\hline Citation & $\begin{array}{l}\text { Journal of Integrative Neuroscience, 3(2), 159-182 } \\
\text { https://doi.org/10.1142/S021963520400049X }\end{array}$ \\
\hline Issue Date & 200406 \\
\hline Doc URL & http:/hdl.handle.net/2115/589 \\
\hline Rights & Copyright (c) 2004 Imperial College Press. The original publication is available at www.worldscinet.com \\
\hline Type & article (author version) \\
\hline Note(URL) & http:/www.worldscinet.comßinßin.shtml \\
\hline File Information & TF.JIN.pdf \\
\hline
\end{tabular}

Instructions for use 
Journal of Integrative Neuroscience

(C) Imperial College Press

\title{
CHAOTIC ITINERANCY AS A MECHANISM OF IRREGULAR CHANGES BETWEEN SYNCHRONIZATION AND DESYNCHRONIZATION IN A NEURAL NETWORK
}

\author{
ICHIRO TSUDA, HIROSHI FUJII*, SATORU TADOKORO, \\ TAKUO YASUOKA, AND YUTAKA YAMAGUTI \\ Department of Mathematics, Graduate School of Science \\ Hokkaido University, Sapporo 060-0810, Japan \\ tsuda@math.sci.hokudai.ac.jp \\ http://www.math.hokudai.ac.jp/ ${ }^{\sim} t s u d a /$ \\ * Department of Information and Communication Sciences \\ Kyoto Sangyo University, Kyoto 603-8555, Japan \\ fujii@bacco.kyoto-su.ac.jp
}

Received 25 June 2003

Accepted 13 December 2003

\begin{abstract}
We investigate the dynamic character of a network of electrotonically coupled cells consisting of class $I$ point neurons, in terms of a finite dimensional dynamical system. We classify a subclass of class $I$ point neurons, called class $I^{*}$ point neurons. Based on this classification, we use a reduced Hindmarsh-Rose (H-R) model, which consists of two dynamical variables, to construct a network model consisting of electrotonically coupled H-R neurons. Although biologically simple, the system is sufficient to extract the essence of the complex dynamics, which the system may yield under certain physiological conditions. The network model produces a transitory behavior as well as a periodic motion and spatio-temporal chaos. The transitory dynamics that the network model exhibits is shown numerically to be chaotic itinerancy. The transitions appear between various metachronal waves and all-synchronization states. The network model shows that this transitory dynamics can be viewed as a chaotic switch between synchronized and desynchronized states. Despite the use of spatially discrete point neurons as basic elements of the network, the overall dynamics exhibits scale-free activity including various scales of spatio-temporal patterns.

Keywords: Gap junction-coupled system; class $I^{*}$ neurons; dynamic cell assembly; chaotic itinerancy; Milnor attractor; metachronal waves; synchronization.
\end{abstract}

\section{Introduction}

Recent findings on cortical dynamic behavior show the presence of specific instability in animal and human brains. This instability produces a transitory behavior between dynamic states. A single time series of the transitory behavior is often observed in laboratory experiments to be "non-stationary" with aperiodic changes between dynamic states. These dynamic transitions between dynamic states may be characterized by the term "transitory dynamics". 
Viewed from the dynamical systems standpoint, the basic language to "interpret" various dynamical behaviors has been geometric attractors and attractor dynamics. For instance, steady states can be represented by fixed point attractors. In a similar way, periodic states may be represented by limit cycles, quasi-periodic states by tori, and chaotic states by strange attractors. These attractors are, however, concepts of low-dimensional dynamical systems, and those terms are evidently inadequate for the description of the much more complex and transitory behaviors mentioned above.

To describe higher-dimensional chaotic behavior, only a few concepts to capture such complex dynamics have been proposed. Among others, hyperchaos [40] is defined to express stationary and complex behavior with multiple positive Lyapunov exponents. Although hyperchaos can provide an important conceptual framework for understanding brain dynamics, it cannot adequately describe such complex dynamics, because the concept is still within the classical framework of attractor. Some authors Tsuda [52], Kaneko [26] and Ikeda [24], on the other hand, proposed "chaotic itinerancy" as a concept to describe transitory behavior that might appear to be "non-stationary" for short periods of observation. Furthermore, the idea of stochastic dynamical systems may be highlighted for understanding brain dynamics [32], because the brain works in very noisy environments. In stochastic dynamical systems, the concept of orbits remains in spite of noisy systems, and is typically clarified in the noise-induced order [33], the stochastic resonance [4], and the stochastic chaos [13].

The aim of the present paper is to interpret cortical transitory dynamics in terms of chaotic itinerancy. Recent discovery of the massive and ubiquitous presence of electrotonic coupling (EC) between fast spiking (FS) (and also among low threshold spiking (LTS)) cells in the neocortex [15, 16, 50], has enabled the formulation of a mathematical model in the search for the presence of chaotic behavior in such electrotonically coupled systems. Our computer simulations of an electrotonically coupled neural system are expected to show a possible mechanism for the background neural activity that underlies the synchronization and the transitory dynamics observed in the brain.

The organization of this paper is as follows. In $\S 2$, we show the characteristics of chaotic itinerancy and discuss its possible mechanisms. In $\S 3$, we describe two classes of neurons and their dynamic characteristics in electrotonically coupled systems, and also show the computation results of our proposed model. In $\S 4$, with numerical analyses, we show the mechanism of the transition between synchronized and desynchronized states exhibited in the model. Section 5 is devoted to discussions on information processing with chaotic itinerancy, and the relation of chaotic itinerancy and dynamic cell assemblies.

\section{Chaotic Itinerancy and its Possible Mechanisms}

In this section, we discuss the concept of chaotic itinerancy. The main characteristics of chaotic itinerancy found in previous studies are summarized as follows (although 
not all characteristics should be required before it is considered present) $[28,29]$ :

(1) the appearance of relatively many near-zero Lyapunov exponents,

(2) slow decay of correlations or time-dependent mutual information,

(3) transitions between relatively low-dimensional states that are called attractor ruins and high-dimensional chaotic states,

(4) non-convergence or extremely slow convergence of zero Lyapunov exponents in some cases, and in some other cases extremely slow convergence of even the largest Lyapunov exponent,

(5) lack of both an attractor-tracing property and a pseudo-orbit tracing property.

The first factor means the appearance of relatively many modes with neutral stability as well as definite stable and unstable modes. The second term indicates that the quantities usually follow a power decay, that results in the appearance of a highly ordered but irregular temporal structure, and hence the appearance of history-dependent transitions. The third represents the characteristic of the transition itself such that it differs from simply chaotic behavior. The transition can be characterized as the transitory dynamics between low-dimensional attractor ruins and high-dimensional chaotic states. The fourth factor expresses the characteristics of the Lyapunov spectra, indicating either the absence of ergodicity or weak ergodicity. A typical chaotic system such as Axiom A diffeomorphism has a pseudo-orbit tracing property. In a system with this property, it is guaranteed that its trajectories can be simulated step by step. Other typical chaotic systems do not possess this property and hence each trajectory cannot be properly calculated in simulations. Even in such cases, there is a possibility of obtaining precise information for the global features of the whole attractor, that is, precise statistical properties. If we obtain correct information concerning the statistical properties of orbits, we will know the system's global behavior through computer simulations and also through laboratory experiments. However, it has been observed that the statistical properties of chaotic itinerancy do not necessarily show information about the overall attractor $[45,46,54]$.

Chaotic itinerancy is thus addressed as a transitory dynamics that might appear to be "non-stationary" in short-time observations. The elemental state for the transition is not represented by a conventional geometric attractor. Therefore, we called the representation of such a state an "attractor ruin". The dynamical orbits reach an attractor ruin, stay in its neighborhood according to a certain distribution of residence times, escape, and again reach the same or another ruin. This transitory dynamics continues without external perturbations. One possible mathematical description of an attractor ruin may be an attractor in Milnor's sense [34]. The geometric attractor which is defined by using the concept of attracting sets, and the Milnor attractor are defined as follows. 
Definition 2.1. Let $M$ be a compact, or at least finite-dimensional, smooth manifold. Let the development of orbits in phase space be given by a continuous map $\phi$ : $M \rightarrow M$. For a set $A_{g}$, the trapping region $S \supset A_{g}$ is defined as the region satisfying $A_{g} \subset \phi(S) \subset S$. A set $A_{g}$ is called an attracting set when $\cap_{i} \phi^{(i)}(S)=A_{g}$, where $\phi^{(i)}$ is the ith iteration of $\phi$, and $\cap_{i}$ denotes infinitely many set intersections. A set $A_{g}$ is called an attractor if it is an attracting set and $\phi \mid A_{g}$ is topologically transitive, meaning that $A_{g}$ cannot be separated into subsets by $\phi$.

According to this definition, any point in a neighborhood of geometric attractor asymptotically approaches the attractor. On the other hand, a Milnor attractor is defined to extend the definition of attractor, taking account of both measuretheoretic and geometric concepts.

Definition 2.2. Let $\rho$ be a measure equivalent to the Lebesgue measure on $M . A$ compact invariant set $A_{M}$ is called a Milnor attractor if the following conditions hold:

a) the basin of attraction $B\left(A_{M}\right)$ of $A_{M}$ has a positive $\rho$-measure, i.e. $\rho\left(B\left(A_{M}\right)\right)>0$;

b) there are no proper closed subset $A_{M}^{\prime}$ satisfying $\rho\left(B\left(A_{M}\right) \backslash B\left(A_{M}^{\prime}\right)\right)=0$.

According to this definition, a Milnor attractor may accompany an unstable manifold, but it attracts orbits with positive measure. Milnor attractors have been studied for chaotic dynamical systems with symmetry [2, 27]. For example, a system of globally coupled identical chaotic oscillators with the same coupling strength is invariant under replacements of elements, and this invariance under this group action gives rise to the invariance under the dynamics. A synchronized state of all oscillators, which has chaos as a base state, forms a chaotic invariant set. In this symmetric case, it turns out that the Milnor attractors appear, probably, associated with a riddled basin, and this situation can allow the occurrence of chaotic itinerancy between partially synchronized states. However, asymmetric cases, like non-equilibrium neural networks [51], can also produce chaotic itinerancy, and thus such symmetry under group action is not prerequisite for the existence of chaotic itinerancy.

\section{Chaotic Itinerancy in Networks of Electrotonically Coupled Class $I^{*}$ Point Neurons}

As explained in detail in previous papers which will be published elsewhere, it has recently been revealed that electrotonic coupling is ubiquitous, even in mammalian neocortex [49], although it had been believed that electrotonic coupling plays a major role only in subcortical systems (e.g., in the inferior olive nucleus or hippocampus) or in invertebrates' neural systems.

Concerning the neuron type, as Alan Hodgkin pointed out [22], two classes of 
neurons (more precisely, excitatory membranes of space-clamped neurons or simply point neurons) exist: class $I$ and class $I I$. The latter may include the unmyelinated giant axon membrane of the squid Loligo, first modeled by Hodgkin and Huxley [23]. In a physiological condition where a current is injected to soma with the strength gradually increasing, class $I I$ point neurons abruptly start firing with a rather high frequency at a critical current strength, and the frequency does not increase much, even if the strength of the input current is greatly increased. This situation may well be attributed to the occurrence of a Hopf bifurcation, if the strength of the input current is regarded as the bifurcation parameter. On the other hand, class $I$ point neurons are characterized by regular repetitive firings with frequencies that vary widely with even slight changes in the strength of the input current. They start firing with an infinitely long period at a critical value of input current strength, and the frequency increases as the strength of the input current increases. Mathematically, this situation could occur if a saddle-node bifurcation structure was imbedded in the neuronal system.

Although the concept of "classes" of neurons does not depend on the number of variables in any model equations, we restrict our arguments to a reduced form with two variables, where the first variable, $V$, may represent the membrane potential, and the second one, $\mathrm{R}$, an activation state of, for example, some potassium channels.

$$
\left\{\begin{array}{c}
C \frac{d V}{d t}=f(V, R)+I \\
\tau_{R} \frac{d R}{d t}=g(V, R) .
\end{array}\right.
$$

In fact, Rinzel [38], Rose and Hindmarsh [42], Wilson [56] and others have proposed that such reduced models extract the "essential dynamics" of space-clamped or point neurons under consideration. In two-variable reduced models, dynamic behavior can be characterized by nonlinearity of the two nullclines corresponding to the two variables. Class $I$ point neurons have $J$ or $U$-shaped nullclines for the $R$ variable, while that for class $I I$ point neurons is essentially a linear nullcline. (It should be noted that the nullcline of a $V$-variable is essentially a graph of cubic function for both classes.)

Within the family of class $I$ point neurons, the Morris-Lecar model, $I_{M L}$ [35], has been known as a mathematical model to study bifurcations of dynamical behavior. It is noted, however, that the Morris-Lecar equation was designed to model barnacle invertebrate neurons, taking into account only the calcium and the rectifying potassium channels, but not intended to model realistic aspects of neocortical neurons. A subclass of the Morris-Lecar model is known to possess saddle-node bifurcations, which allows the presence of a homoclinic orbit at the bifurcation point $[19,20,25,39]$.

Another example of class $I$ point neurons is the one originally considered by Connor and others $[6,7,8,9]$. They included an effect of the transient potassium 
current, called the $A$-current, resulting in the Hodgkin-Huxley-type equations with six dynamical variables. The reduction of the Connor equations to a two-variable model was presented by Rose and Hindmarsh [42], and Wilson [56]. The class $I$ point neuron models of Connor type, $I_{C}$, have essentially quadratic nullclines of $R$ variable. As the injected current $I$ increases from the resting state, the saddle-node bifurcation appears, yielding an action potential with an infinitely small frequency at the critical regime. After a pair annihilation of saddle and node, a narrow "channel" appears in the phase space between the two nullclines. The presence of this narrow channel characterizes the class $I$ point neurons of Connor type, $I_{C}$, as compared with the Morris-Lecar model, $I_{M L}$, together with the differences in bifurcation structure of the two models.

While the orbit is in the narrow channel, the point neurons undergo a subthreshold polarized state. The residence time in the channel depends on the width of the channel, and the channel width depends sensitively on the input current level. Thus, the firing frequency of individual point neurons of class $I_{C}$ possesses a sensitive dependence on the fluctuations of input current near the saddle-node bifurcation point.

The class $I$ reduced models of Connor type $I_{C}$ may further be classified into two subclasses: $I_{C}{ }^{(a)}$ and $I_{C}{ }^{(b)}$, according to whether the remaining equilibrium point is an unstable spiral, $I_{C}{ }^{(a)}$, or an unstable node, $I_{C}{ }^{(b)}$, in the parameter range where the narrow channel exists. An example of the class $I_{C}{ }^{(a)}$ point neuron model can be given as follows, which is a modified version of Wilson model of class $I_{C}^{(b)}[57]$ :

$$
\begin{aligned}
& \left\{\begin{array}{l}
f(V, R)=-m_{\infty}(V)\left(V-E_{N a}\right)-g_{K} R\left(V-E_{K}\right) \\
g(V, R)=-R+R_{\infty}(V) .
\end{array}\right. \\
& m_{\infty}(V)=5.36+17.04 V+16.9 V^{2}, \\
& R_{\infty}(V)=1.29 V+0.53+3.3(V+0.18)^{2}, g_{K}=11.0 \\
& \quad E_{N a}=0.48, E_{K}=-0.95, C=1.0, \tau_{R}=2.4
\end{aligned}
$$

Our main interest here is the study of the system-level behavior of a network of electrotonically coupled cells consisting of class $I$ point neurons, rather than the dynamics of an isolated single neuron, because we believe that the spatio-temporal activity of neural assemblies at the mesoscopic level is responsible for higher-level functions such as memory, perception and cognition.

The network of electrotonically coupled cells can be described by:

$$
\begin{aligned}
C \frac{d V_{j}}{d t} & =f\left(V_{j}, R_{j}\right)+g_{G J} \sum_{n b_{j}}\left(V_{n b_{j}}-V_{j}\right)+I_{j} \quad\left(n b_{j} \in \text { coupled neigbor cells }\right) \\
\tau_{R} \frac{d R_{j}}{d t} & =g\left(V_{j}, R_{j}\right) .
\end{aligned}
$$


Here, the electrotonic coupling is assumed to take the form of linear diffusions in the $V$-variable (see, for example, [47]). The strength of this electrotonic coupling is denoted by $g_{G J}$. The summation is taken over the "neighboring" point neurons that are coupled with the other cells concerned, although this is not necessarily a neighborhood in the Euclidean space, as the couplings may exist with cells placed far from the neuron's soma.

For the case of all-to-all electrotonic couplings with the Morris-Lecar models, Han et al. $[19,20]$ reported the presence of chaotic alterations of synchronized and desynchronized states in a very narrow parameter region. This chaotic behavior occurs in a neighborhood of a critical regime of bifurcation, at the bifurcation point of which a homoclinic orbit appears; hence the name homoclinic bifurcation. It may be a kind of chaotic itinerancy, although the chaotic states in this model are in an unrealistic membrane potential range and a mechanism for such chaotic states has not been clarified.

As a preliminary work, we investigated nearest-neighbor couplings for the MorrisLecar neurons, $I_{M L}$. This case also produces chaos but again in very narrow parameter regions. Among class I point neurons, we could not find any chaotic behavior in a similar situation of nearest-neighbor couplings for either the six-variable Connor point neuron, or for $I_{C}{ }^{(b)}$ point neurons. In contrast, point neurons of class $I_{C}{ }^{(a)}$ change to exhibit chaotic behavior with large fluctuations in both phase and amplitude when electrotonically coupled. Here, they show perfectly regular firings when isolated. Thus a single cell's activity is represented by a limit cycle oscillator. This occurs near the saddle-node bifurcation point of the injection current, but with a rather large range of the coupling strength. The above observation for class $I_{C}{ }^{(a)}$ behavior is regarded as a special case of the class $I^{*}$, that is, a point neuron class defined in a more abstract setting.

Let $I^{*}$ denote a subclass of class $I$ point neurons, in which there is a certain interval of injected current, $I$, such that the following conditions hold simultaneously:

1. the presence of a family of limit cycles with periods that go to infinity at the saddle-node bifurcation point;

2. the presence of a narrow channel; and

3. the presence of an unstable spiral inside the closed orbit described in 1.

It should be noted that the presence of a saddle-node bifurcation is not a sufficient condition for a cell to be of class $I^{*}$, although it is a natural situation in most models. We expect that the class $I^{*}$ point neurons exhibit chaotic behavior in some parameter regions when electrotonically coupled.

The subclass $I_{C}{ }^{(a)}$ of the Connor type satisfies these "essential conditions", while the Morris-Lecar model $I_{M L}$ does not, because it does not possess a property corresponding to the above second condition. The class $I$ model of Rose and Hindmarsh and that of Wilson do not satisfy the third condition, and thus do not belong to $I^{*}$.

It is not difficult to construct a simplified model of class $I^{*}$ rather than $I_{C}{ }^{(a)}$. 
For instance, a piecewise-linear model can be in class $I^{*}$. In the present paper, the following one-parameter $(\mu-)$ family of two-variable models, which are of class $I^{*}$, is studied as the basic model. This model can be reduced from the two-variable Hindmarsh-Rose model [21] by simple coordinate transformations and reduction of the parameters. Hindmarsh and Rose [21] introduced one slow variable to the two-variable model to produce the bursts. For our present purpose, we neglect this slow variable. Although, in this respect, our present model is a special case of the Hindmarsh-Rose model [21], the model exhibits a whole bifurcation structure associated with the appearance of saddle-node and Hopf bifurcations that the class $I^{*}$ possesses. Thus, our model possesses necessary and sufficient features to express class $I^{*}$ point neurons, and hence the essential minimum. This minimum model for the Connor equations may have a similar relation to the Hindmarsh-Rose model as the FitzHugh-Nagumo model has to the Hodgkin-Huxley equations in a mathematical sense, but not in a physiological sense.

$\mu$-model: (class $I^{*}$ )

$$
\left\{\begin{array}{l}
\frac{d x}{d t}=-y-\mu x^{2}\left(x-\frac{3}{2}\right)+I \\
\frac{d y}{d t}=-y+\mu x^{2} .
\end{array}\right.
$$

In fact, the $\mu$-model yields abundant chaotic behavior and chaotic itinerancy in its coupled systems. We model electrotonic coupling following Schweighofer et al. [47], which take essentially a diffusion-like form with a mild nonlinearity. In the present simulation, we assume linear diffusion-type couplings in the $\mu$-model. Corresponding to the Neumann type of boundary condition in a continuous space model, we adopt a free boundary condition in the present coupled system.

The overall network is modeled as follows:

\section{Electrotonic coupling of $\mu$-models}

$$
\begin{aligned}
& \text { For } i=1, \ldots, N \text {; } \\
& \left\{\begin{array}{l}
\frac{d x_{i}}{d t}=-y_{i}-\mu x_{i}{ }^{2}\left(x_{i}-\frac{3}{2}\right)+I_{i}+J_{i} \\
\frac{d y_{i}}{d t}=-y_{i}+\mu x_{i}{ }^{2}
\end{array}\right.
\end{aligned}
$$

where the $I_{i}$ 's represent injected background currents (which are assumed to be constant in time and space in this simulation), and the $J_{i}$ 's represent the currents induced by electrotonic couplings: 


$$
\begin{aligned}
J_{i} & =g_{G J} \sum_{n b_{i}}\left(x_{n b_{i}}-x_{i}\right) \quad\left(n b_{i} \in \text { coupled neigbor cells }\right) \\
& =\left\{\begin{array}{l}
g_{G J}\left(x_{2}-x_{1}\right)(i=1) \\
g_{G J}\left(x_{i+1}+x_{i-1}-2 x_{i}\right)(i=2, \ldots, N-1) \\
g_{G J}\left(x_{N-1}-x_{N}\right)(i=N) .
\end{array}\right.
\end{aligned}
$$

Here, $g_{G J}$ is a coupling constant that is assumed to be identical for all connections in the present simulation.

Figure 1(a) shows a time series of 30 coupled $\mu$-model neurons exhibiting spatio-temporal chaos. The degree of instability of the orbits produced by this $60-$ dimensional dynamical system can be estimated by the Lyapunov exponents that define orbital instability [36]. The exponents can be calculated as an expanding or a contracting rate in each direction of orthonormal vectors in the time development of orbits, and thus they can be represented by a long-time average of the eigenvalues of the matrix formed by multiplication of Jacobian matrices [11, 44]; hence the name multiplicative ergodic theory [36]. Several methods could be used for numerical simulations or experimental data analysis, but the best method to use depends on the norm adopted [3]. A set of Lyapunov exponents is called a Lyapunov spectrum. According to a conventional definition of chaos, the presence of positive Lyapunov exponents indicates chaos.

The Lyapunov dimension that can approximate the Haussdorf dimension of the obtained chaotic attractor is 34.158 in the case of the spatio-temporal chaos shown in Fig. 1. This dimensionality indicates the effective dimension of an attractor in 60-dimensional vector space. On the other hand, the topological dimension, i.e., the dimension of the support of the attractor, is only 20 in the present case, that is the total number of non-negative Lyapunov exponents. This dimension gap [41] $(34.158-20>1)$ stems from the large number of negative Lyapunov exponents with relatively small absolute values. A large dimension gap like this implies that an attractor is distributed over a wide range in phase space.

Figure 1(b) shows a time series of a contour map in which the colors denote the level of amplitude of the $x$-variable (namely, the membrane potential) of the neurons. Thus, it indicates the spatio-temporal organization of neural activity. Many cell assemblies, each of which yields a different metachronal wave, are temporarily formed, giving rise to a reorganization of cell assemblies. Here, a metachronal wave means a wave formed by neural activity with orderly phase shifts. A metachronal wave is observed in the movement of a centipede and that of cilia and flagella. The change of behavior in this simulation is highly complex, that is, it demonstrates chaotic changes of cell assemblies formed by metachronal waves. We call this complex form a metachronal assembly. 
(a)

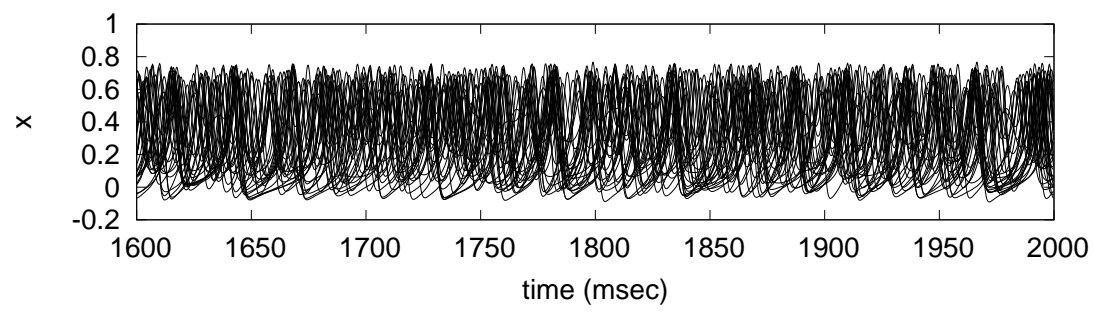

(b)

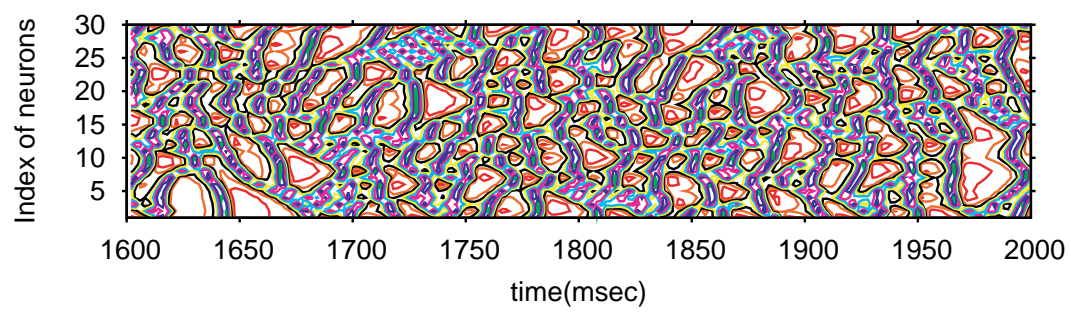

Fig. 1. Dynamic behavior of a network of $\mu$-model neurons with electrotonic couplings. Parameters are as follows: $\mu=1.65, \mathrm{I}=0.005, g_{G J}=0.05$. All present simulations for integration of the differential equations are performed by using the fourth order of Runge-Kutta-Gill method with time increment $0.02(\mathrm{~ms})$.

(a) Superimposed time series of the membrane potentials of 30 coupled $\mu$-model neurons. The abscissa denotes time and the ordinate the amplitude of the potential denoted by the $x$-variable.

(b) Spatio-temporal patterns represented by a contour map. Color denotes the level of amplitude of the $x$-variable (namely, the membrane potential) of the neurons.

If a metachronal wave appears as a certain definite state, then it could be represented as an attractor. Actually, to a first approximation, in a moving coordinate, $x-v t$, this wave could be viewed as a fixed-point attractor of the motion. The transition between metachronal waves and spatio-temporal chaos may be viewed as a transition between low-dimensional Milnor attractors and high-dimensional chaotic states. In fact, chaotic itinerancy between metachronal waves is observed in this model. At least, the four factors (1)-(4) described in $\S 2$ are confirmed. A typical behavior is shown in Fig. 2 by the contour map and the time series of the $x$ variables. Similar metachronal assemblies appear frequently, and they are not periodic but chaotic in such a way that spatio-temporal patterns become quite different soon after a very close metachronal assembly appears. The Lyapunov dimension is 8.045, and the topological dimension is 5 for the chaotic itinerancy shown in Fig. 2. Compared with fully developed chaos in Fig. 1, the dimensionality becomes low, reflecting the appearance of the relatively low-dimensional ordered motion that forms metachronal waves. 


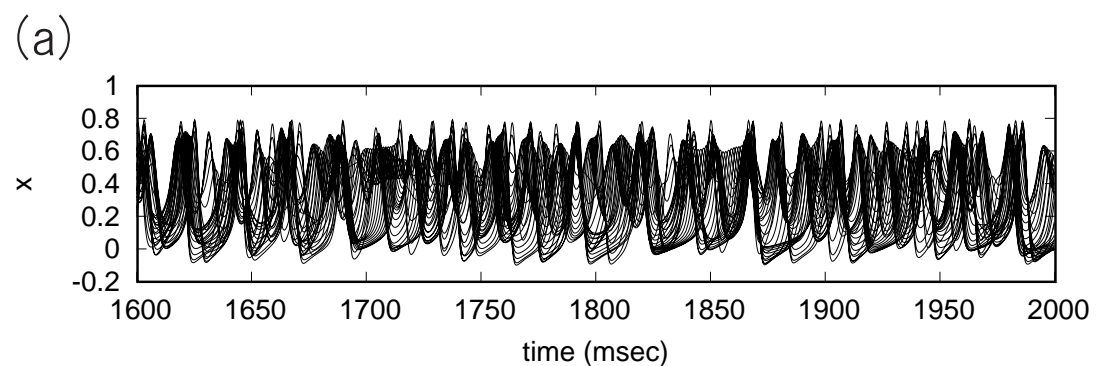

(b)

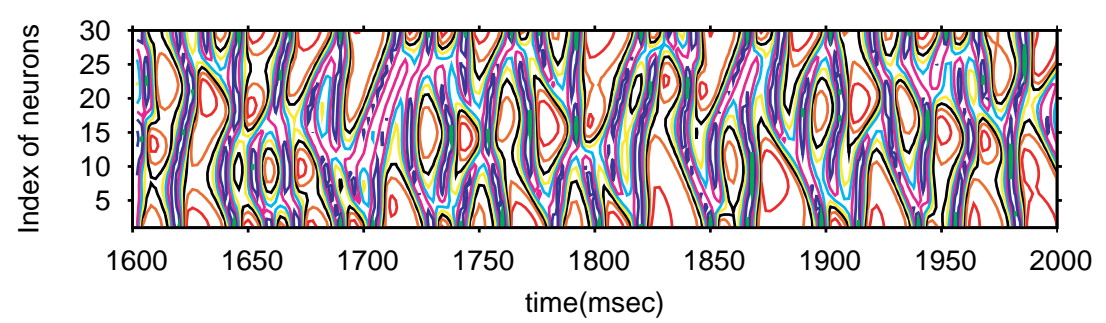

Fig. 2. A typical chaotic itinerancy between metachronal waves. $\mu=1.65, \mathrm{I}=0.005, g_{G J}=0.5$.

(a) Superimposed time series of the membrane potentials of 30 model neurons.

(b) Spatio-temporal patterns by a contour map. Color denotes the level of amplitude of the $x$-variable (namely, the membrane potential) of the neurons.

Figure 3 shows the chaotic alteration of synchronized and desynchronized states, here the transitory dynamics may provide a mechanism for cortical transient synchronization. This situation is realized when in addition to a relatively strong coupling of point neurons, a single $I^{*}$ point neuron exhibits longer periods of oscillations of spikes than are seen in fully developed turbulence. This stems from a slow motion in the vicinity of the original saddle, that is, in the presence of a narrow channel in phase space, where the saddle itself disappears but its ruin remains. This can be controlled by an injected current, but not necessarily by the ion channels' intrinsic character. The chaotic alteration between synchronized and desynchronized states is also described as chaotic itinerancy, because this behavior satisfies at least characteristics (1)-(4) of chaotic itinerancy. Very slow convergence of the largest Lyapunov exponent (see also [54] as well as [29]) is also observed in the present condition, which is shown in Fig. 3 (c), although it is not clear whether it is so "nonstationary" as to satisfy factor (5). Highly ordered structures in a complex transitory dynamics can also be estimated by the decay of correlations, which is depicted in Fig. 3 (d). 
(a)
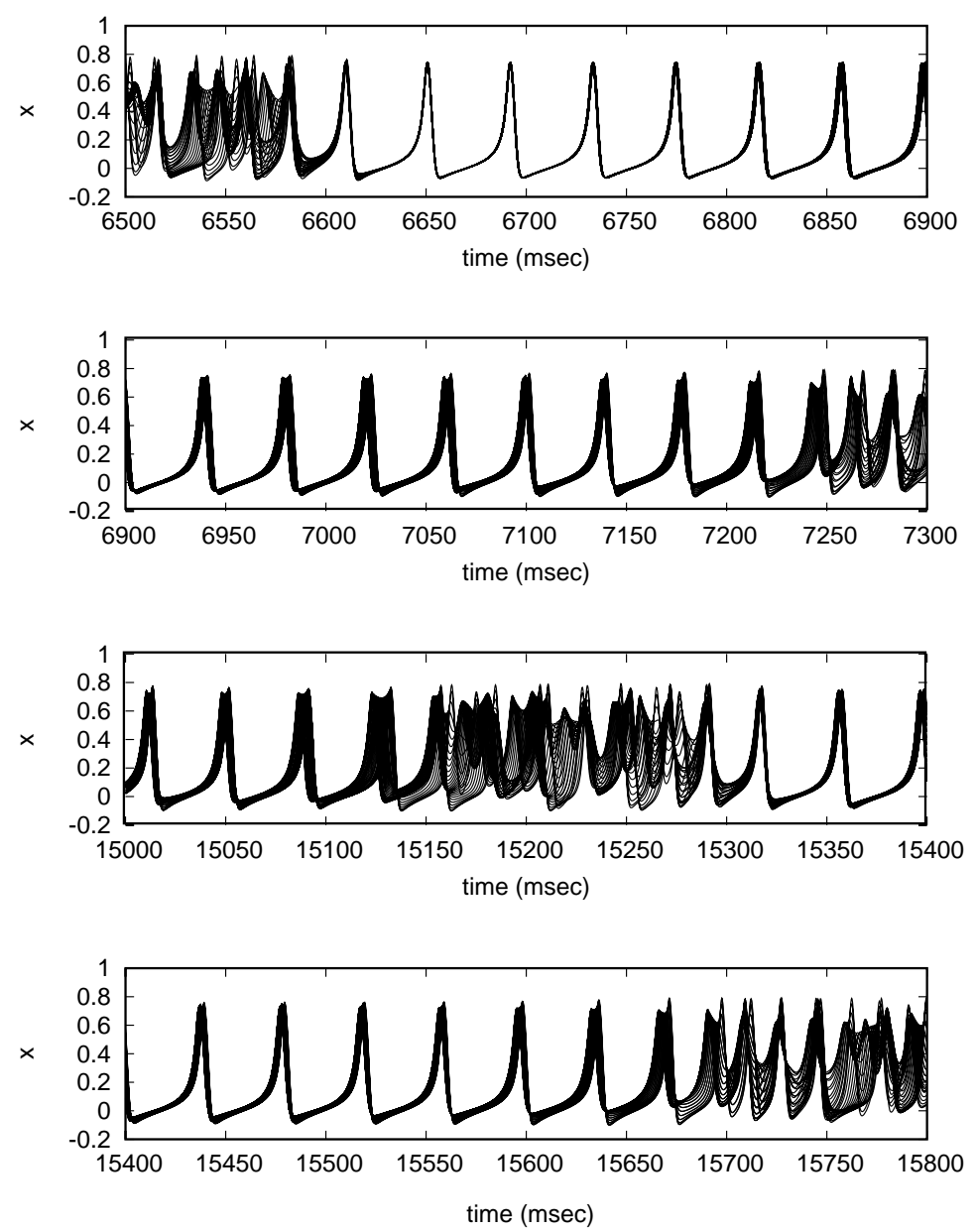
(b)
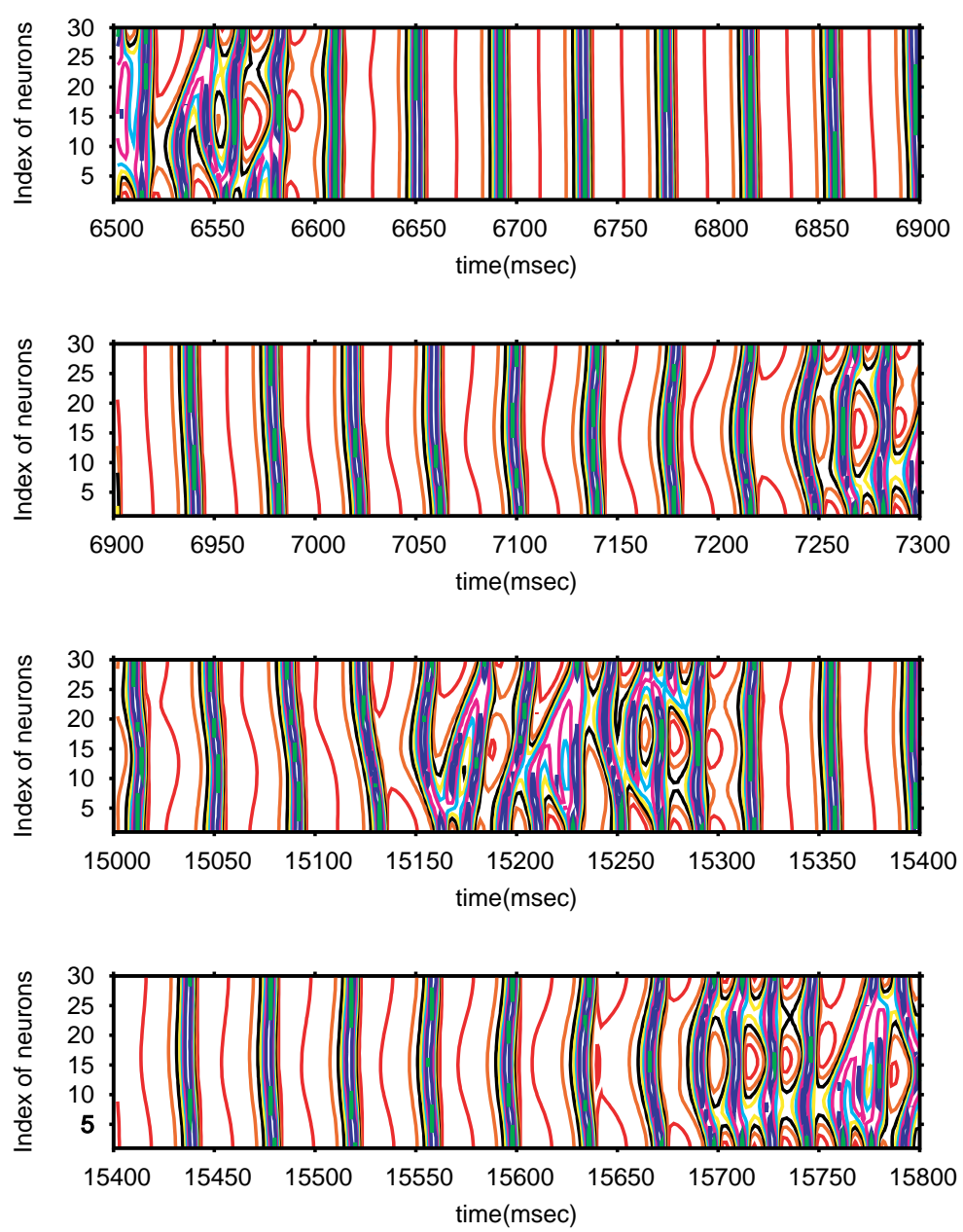

(c)

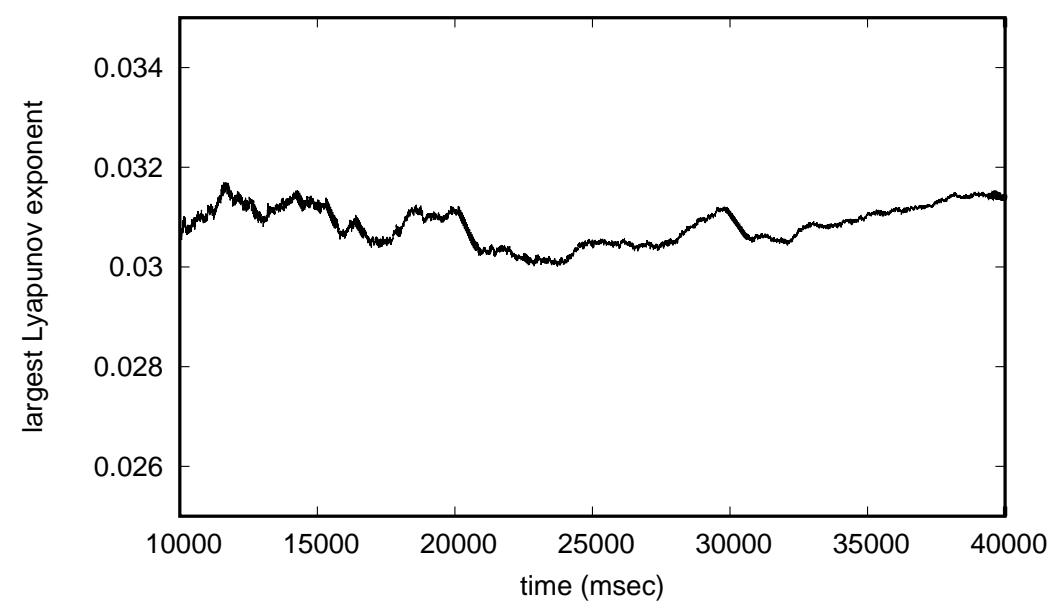


(d)
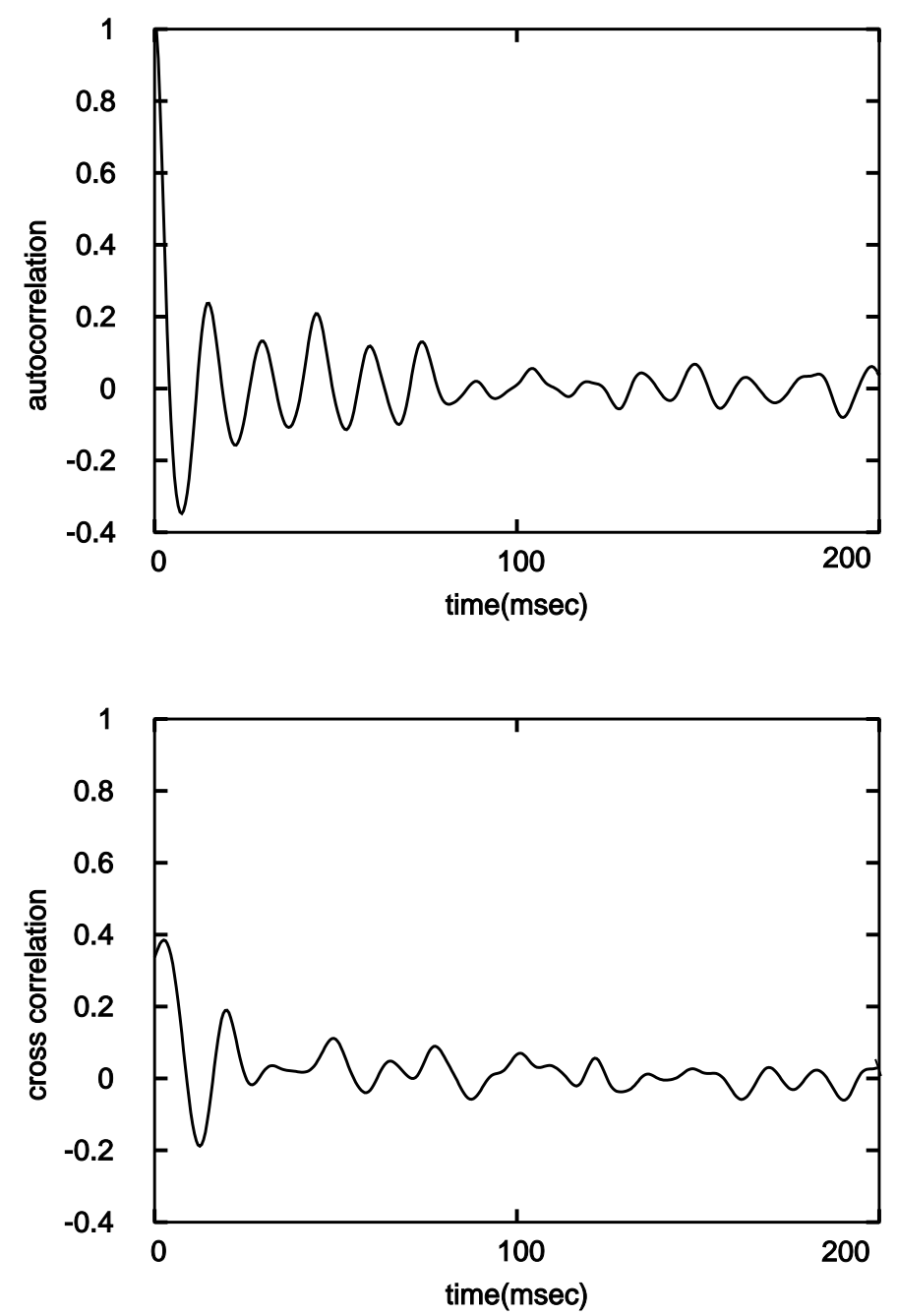

Fig. 3. A typical chaotic alteration of synchronized and desynchronized states. This shows another typical chaotic itinerancy observed in the model. $\mu=1.65, \mathrm{I}=0.005, g_{G J}=0.83$.

(a) Superimposed time series of the membrane potentials of 30 model neurons. The upper two time series are depicted during $t=6,500-7,300 \mathrm{~ms}$, and the lower two during $t=15,000-15,800 \mathrm{~ms}$.

(b) Spatio-temporal patterns by contour map. The upper and the lower figures correspond to those of (a).

(c) The fluctuations of the largest Lyapunov exponent.

(d) The auto-correlation of the first neuron (above) and the cross-correlation between the first and the 15 th neurons (below).

Even the highly developed spatio-temporal chaos exhibited in Fig.1 contains ordered structures that are made of metachronal assemblies with various spatiotemporal scales. Because the extreme of an infinite velocity in metachronal waves 
is viewed as a completely synchronized state, we can describe a transition between synchronized and desynchronized states as a continuation of the transition between metachronal waves. The former transition has actually been observed in physiological experiments. Thus we expect chaotic itinerancy between partially synchronized states or between various metachronal waves. In general, nearest-neighbor couplings of the diffusion type easily yield metachronal waves, and a complete synchronization is usually unstable. A wider range of couplings than the nearest-neighbor couplings, yields states close to synchronization more easily. The increase of the size of such a range tends to bring about the increase of the spatial size of partial synchronizations, i.e., synchronized assemblies.

\section{The Mechanism of Chaotic Itinerancy Between Synchronized and Desynchronized States}

By studying further the dynamic behavior in electrotonically coupled $\mu$-models, we clarify a mechanism of chaotic itinerancy between the synchronized and desynchronized states. We calculated the transverse Lyapunov exponents in a subspace transversal to the subspace in which the activity of all point neurons are completely synchronized, where the activity is represented by an individual limit cycle oscillator. Two positive transverse Lyapunov exponents appear; their situation indicates that this all-synchronization state (denoted by $S_{A}$ ) is unstable in its transverse directions. This state is stable only within the two-dimensional subspace, $M_{\text {all-syn }}$, formed by $x_{1}=x_{2}=\ldots x_{N}$ and $y_{1}=y_{2}=\ldots y_{N}$. Because this space is measure-zero in a whole space, the all-synchronization state, $S_{A}$, is not even a Milnor attractor, but simply a saddle. However, we often observe the re-entering orbits in a neighborhood of $S_{A}$ and hence the repeated appearance of a quasi all-synchronization state, which is described by the synchronized state of all oscillators within very small phase shifts.

We further investigate which components of motion destabilize the allsynchronized state, $S_{A}$, focusing on positive transverse Lyapunov exponents. At most two such exponents appear. These two exponents correspond to two kinds of metachronal waves, which often appear in the present model as fundamental components of the complex behavior of spatio-temporal chaos. One kind of metachronal wave consists of two different metachronal waves, denoted by $S_{u}{ }^{+}$and $S_{u}{ }^{-}$. The difference of these two waves is only in the direction of phase shift, i.e., opposite directions to each other, which is due to the symmetry of the system (see Fig. 4 (a)). The other kind of metachronal wave, denoted by $S_{S}$, has the symmetric form of phase shift in both directions from the center, where the center is a neural element placed in the middle of the network in the present Neumann boundary condition (see Fig. 4 (a)).

The scenario of the chaotic transition between synchronized and desynchronized states is sketched as follows. We describe the dynamic behaviors on the Poincaré section, $\Sigma_{i} x_{i} / N=0.2$, which clarifies the discussion based on dynamical systems' 
theory. To show the correspondence of dynamical systems terminology to realistic patterns, the corresponding spatio-temporal patterns are given in Fig. 4 (a).

Two saddles, $S_{A}$ and $S_{S}$, exist on the section, representing an all-synchronized periodic state and a metachronal wave, respectively. There are also two stable fixed points, $S_{u}{ }^{+}$and $S_{u}{ }^{-}$, representing the other kind of metachronal waves; their appearance depends on the initial conditions. During the initial period, the fixed points, $S_{u}{ }^{+}$and $S_{u}{ }^{-}$, become unstable simultaneously via Hopf bifurcations. Thus, two limit cycles are generated on the section, indicating a genesis of two tori in a whole space, the appearance of which depends on the initial conditions. These states represent modulated metachronal waves. Such tori merge in a neighborhood of the all-synchronized state, $S_{A}$, and the state of the metachronal wave, $S_{S}$, and then a large torus enclosing $S_{u}{ }^{+}$and $S_{u}{ }^{-}$in an eight-shaped fashion appears. Therefore, this large torus represents a quasiperiodic transition between the all-synchronized state and the two kinds of metachronal waves. The bifurcations at the next stage are complicated: at least a region, denoted by $X_{S}$, exists, inside which toric or chaotic motion enclosing $S_{u}{ }^{+}$and $S_{u}{ }^{-}$occurs. This represents a toric or a chaotic transition between the all-synchronized state and the different kinds of metachronal waves, $S_{A}$, $S_{S}, S_{u}{ }^{+}$and $S_{u}^{-}$. Next, large chaotic orbits, denoted by $C_{C}$, are generated, due to an interior crisis, with orbits starting near the metachronal wave state, $S_{S}$. These orbits experience a large excursion, and come back near the all-synchronized state, $S_{A}$.

At the next stage of bifurcation, further complex and itinerant transitions are induced, where the objects consisting of the all-synchronization state, the different kinds of metachronal waves, and the large chaotic orbits, depicted in Fig. 4 (a), can be Milnor attractors (or attractor ruins). This situation can be described by chaotic itinerancy, in the sense of chaotic transitions between Milnor attractors, each consisting of an all-synchronization state, different kinds of metachronal waves, and large chaotic orbits. A typical trajectory of such a chaotic itinerancy is shown in Fig. 4 (b), drawn in the projection to the space $x_{1}-x_{15}$ of the Poincaré map with the Poincaré section, $\Sigma_{i} x_{i} / N=0.2$, and also a time series of the voltage of the first neural element, where $N=30$. Stagnant motion near $x_{1}=0.2$ indicating an all-synchronized state, $S_{A}$, is clearly seen as well as the chaotic or toric states on $X_{S}$, and a large chaotic state, $C_{C}$. 
(a)

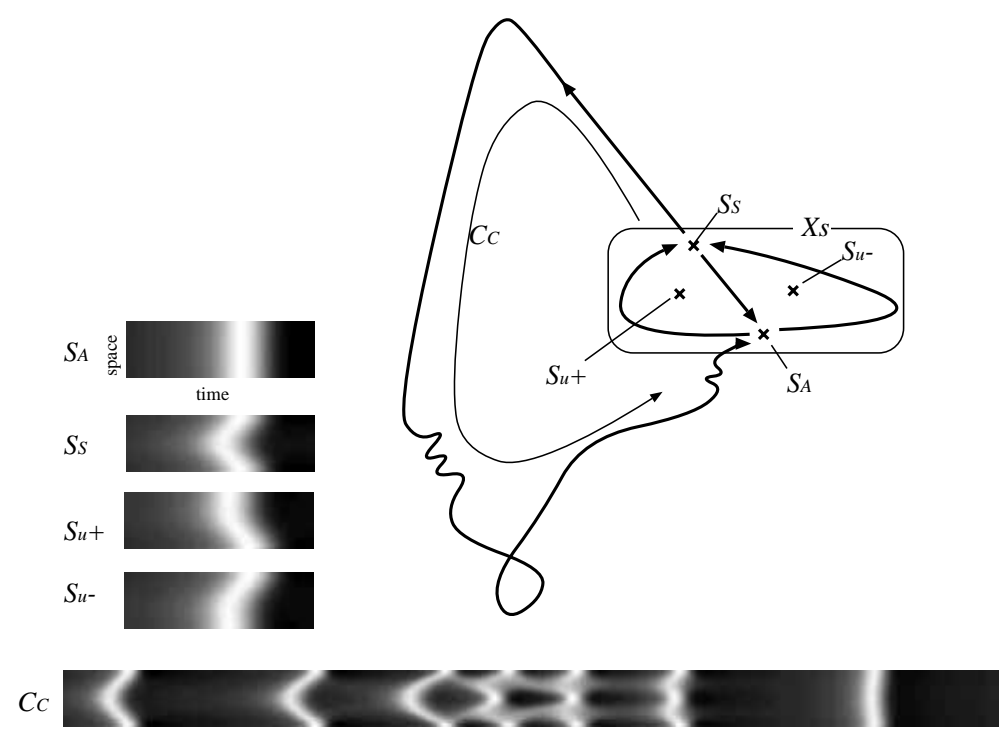

(b)
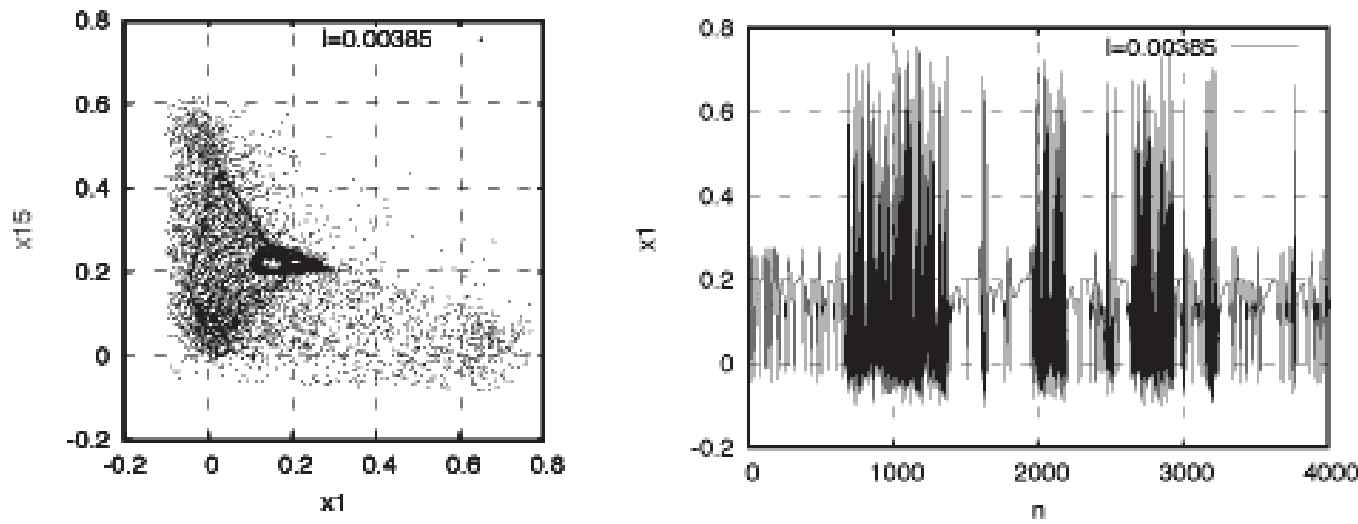

Fig. 4. A fundamental structure of dynamic states for the transition between synchronized and desynchronized states.

(a) Various dynamical states on the Poincaré section, $\Sigma_{i} x_{i} / N=0.2$, where $N=30$, yielding the transition are schematically drawn, together with the corresponding actual spatio-temporal patterns to the dynamical states. The spatio-temporal pattern shown in the lowest inset in the figure can be represented by a Milnor attractor for the present chaotic itinerancy. See details in the text.

(b) Typical trajectories in the projection to $x_{1}-x_{15}$ of a Poincaré map with the Poincaré section, $\Sigma_{i} x_{i} / N=0.2$, where $N=30$, and also a corresponding time series of the voltage of the first neuron, $x_{1}$, is depicted in the case of injection current $I=0.00385$. Here, time $n$ is in discrete time steps, which measure a trajectory on the Poincaré section. 


\section{Summary and Discussions}

We investigated electrotonically coupled $I^{*}$ point neurons, a subclass of class $I$ point neurons, to clarify a mechanism underlying cortical synchronization and its transitory dynamics, in particular, an irregular change between synchronized and desynchronized states. As a model of class $I^{*}$ point neurons, we used a one-parameter family of $\mu$-models. This model possesses a dynamic character such that networks with electrotonic couplings easily yield chaotic behaviors even though each produces a periodic activity.

We found, in the present paper, ordered but ever-changing dynamic behaviors of neural assemblies, accompanying states of different kinds of metachronal waves and all-synchronization. The findings allow us to provide a dynamical interpretation of cortical transitory dynamics in terms of chaotic itinerancy. The behaviors obtained may explain the cortical mechanism of "chaotic" and "nonstationary" transitions between synchronized and desynchronized states that have been observed in cortical activity $[12,13,17,30,31,48]$ and in model neural assembly systems with some theoretical frameworks [5, 19, 20, 25, 37, 55].

Our model simulations are based on class $I^{*}$ point neurons, but such electrotonically coupled cells show dynamic behaviors reflecting the appearance of macro-variables, i.e., the order parameters. This is clearly seen in Fig. 5. The coarse-grained activity obtained by various scales of ensembles yield quite similar spatio-temporal patterns, which give rise to a similar decay process of auto- and cross-correlations between single neurons activity and also between coarse-grained activity. This implies that various scales of neural assemblies are formed in the network, and this formation of macroscopic ordered motion does not depend on the details of a single cell's feature. This character of independence from single-cell's details, perhaps, stems from chaotic instability inherent in the system.

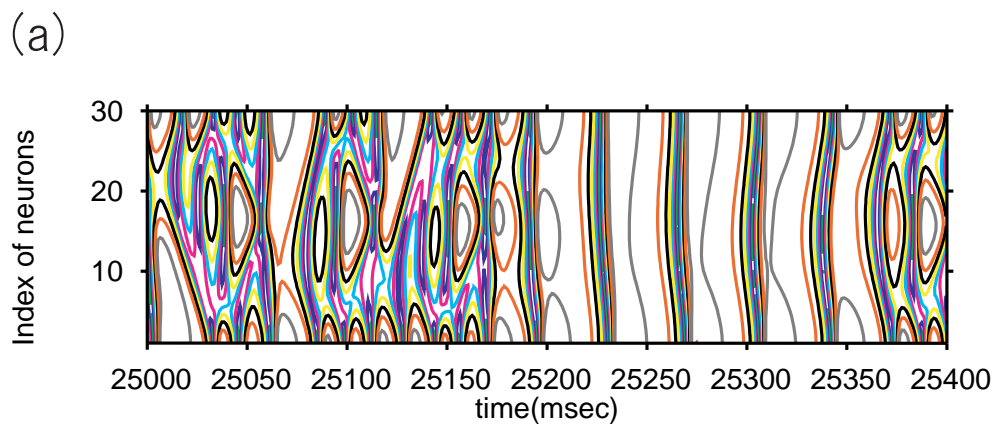


(b)

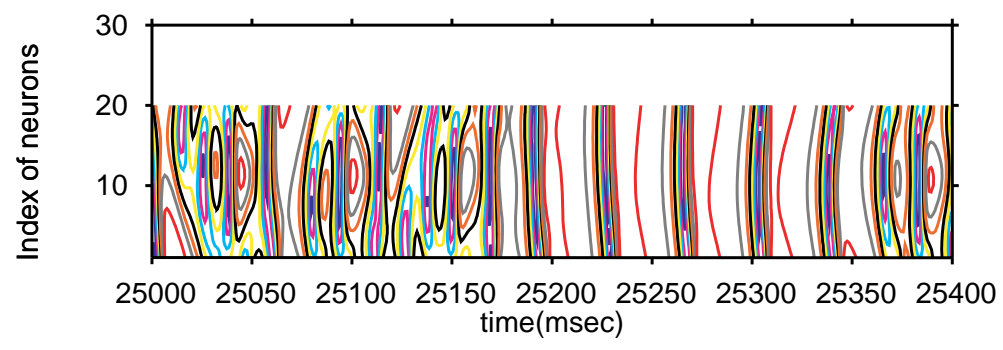

(c)

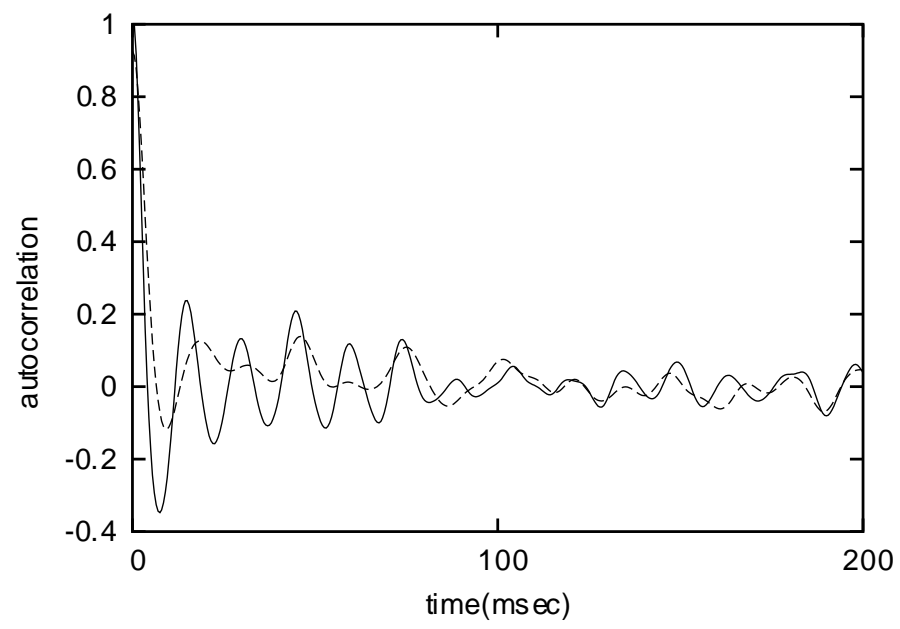

(d)

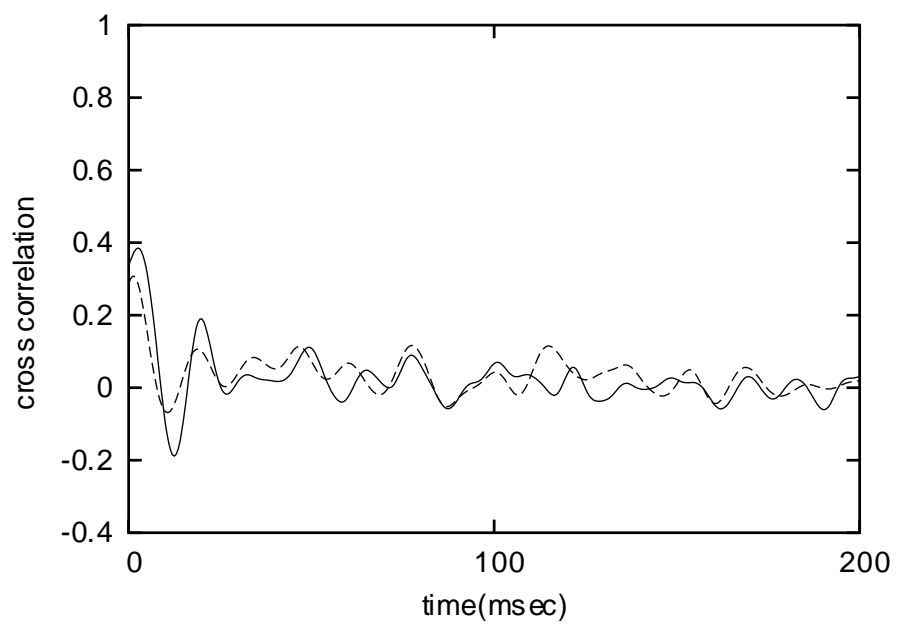


Fig. 5. Scale-free behaviors of spatio-temporal patterns. $\mu=1.65, \mathrm{I}=0.005, g_{G \mathrm{~J}}=0.83$.

(a) Original spatio-temporal dynamics represented by a contour map. Different time interval is shown in the same parameter as in Fig. 3.

(b) Coarse-grained spatio-temporal dynamics represented by contour map. Each dynamics in each unit indicates activity averaged over $L$ model neurons. Here $L=10$. These coarse-grained patterns are essentially the same as the spatio-temporal patterns obtained from a single neuron's activity shown in (a).

(c) Auto-correlations in the case of coarse-graining show the same characteristics as those in the original patterns. Both cases of $L=0$ (original, denoted by a solid line), and 10 (a dotted line) are overlaid.

(d) Cross-correlations between the 1 st and the 15th units. Cross-correlations show the same tendency as auto-correlations. The original is denoted by a solid line and a coarse-grained one $(\mathrm{L}=10)$ by a dotted line.

Metachronal waves can effectively carry the information that could be input into local sites. Chaotic itinerancy between assemblies of metachronal waves may play a role in creating new local assemblies, which may be components [43], that is, the basic format of the brain [55], and transmit information between assemblies by linking these components. By this mechanism of creation of shared information, the reorganization of assemblies may proceed smoothly [53].

As for the chaotic transition between synchronization and desynchronization, it may also be related to the binding problem through synchrony $[10,18,55]$. Inspired by the von der Malsburg theory [55], Aertsen et al. [1], and Fujii et al. [14] have discussed some aspects of dynamic cell assemblies. They proposed that feature bindings could be formed by functional couplings via spike coincidence. Our present results show an alternative possibility; that chaotic itinerancy appears to be associated with dynamic changes of cell assemblies, and thus feature bindings may not necessarily be established by spike coincidence but possibly by chaotic behavior of background FS (fast spiking) interneurons. Here, it should be noted that chaotic fluctuations of inhibitory interneurons, say of FS cells in cortical layers, are not directly reflected in local field potentials. Rather, the inhibitory influence of FS cells on pyramidal cells affects the fluctuations of membrane potentials of the pyramidal cells, and then those fluctuations may cause dynamic changes such as the transitory and synchrony dynamics of local field potentials or EEGs. Recently, Raffone and van Leeuwen [37] demonstrated chaotic itinerancy-like transitions between relatively synchronized and desynchronized states of a layered associative network with three-variable Hindmarsh-Rose neurons [21]. Raffone and van Leeuwen showed in a model study how a flexible feature binding could occur in neural assemblies. Because our present model exhibits various transitory synchronizations under constant inputs, it may provide a dynamic basis for such a flexible feature binding.

Another important open question is whether cortical FS neurons possess 
class $I^{*}$ nature. With these conditions assumed, we propose the following hypothesis:

Chaotic and "non-stationary" changes between synchronization and desynchronization that have been observed in experiments are based on itinerant motion that may be generated by electrotonically coupled point neuron systems. This itinerant motion can be described by the chaotic itinerancy obtained in this paper.

\section{Acknowledgments}

The authors would like to express their special thanks to the other members of the Dynamic Brain Group ("Gang of Five"), Minoru Tsukada, Shigetoshi Nara, and Kazuyuki Aihara. We would also like to thank Walter Freeman for his stimulating discussions on the cortical synchronization and helpful comments. The first author (I.T.) was partially supported by Grant-in-Aid no. 12210001 on priority areas (C), Advanced Brain Science Project, Grant-in-Aid no.15650033 for Creative Scientific Research and also supported by Grant-inAid for formation of COE, while the second author (H.F.) is supported by the Advanced and Innovational Research Program in Life Sciences, both from Ministry of Education, Culture, Sports, Science and Technology, the Japanese Government. Yutaka Yamaguti is partially supported by Research Fellowships of The Japan Society for The Promotion of Science for Young Scientists.

This paper is dedicated to the late Gen Matsumoto.

\section{References}

[1] Aertsen, A., Erb, M., and Palm, G., Dynamics of functional coupling in the cerebral cortex: an attempt at a model-based interpretation, Physica D 75:103-128, 1994.

[2] Ashwin, P. and Swift, J., The dynamics of $\mathrm{n}$ weakly coupled identical oscillators, $J$. Nonlinear Sci. 2:69-108, 1992.

[3] Barna, G. and Tsuda, I., A new method for computing the Lyapunov exponents, Phys. Lett. A, 175:421-427, 1993.

[4] Benzi, R., Sutera, A. and Vulpiani, A., The mechanism of stochastic resonance, J. Phys. A, 14:L453-457, 1981.

[5] Breakspear M., Terry, J. R., and Friston, K. J., Modulation of excitatory synapse coupling facilities synchronization and complex dynamics in a nonlinear model of neuronal dynamics, Network-Comp. Neural., 14:703-732, 2003.

[6] Connor, J. A. and Stevens, C. F., Inward and delayed outward membrane currents in isolated neural somata under voltage clamp, J. Physiol., 213:1-19, 1971.

[7] Connor, J. A. and Stevens, C. F., Voltage clamp studies of a transient outward membrane current in gastropod neural somata, J. Physiol., 213:21-30, 1971.

[8] Connor, J. A. and Stevens, C. F., Prediction of repetitive firing behaviour from voltage clamp data on an isolated neurone soma, J. Physiol., 213:31-53, 1971.

[9] Connor, J. A., Walter, D. and McKown, R., Neural repetitive firing: modifications of 
the Hodgkin-Huxley axon suggested by experimental results from crustacean axons, Biophys. J., 18:81-102, 1977.

[10] Eckhorn, R., Bauer, R., Jordan, W., Brosch, M., Kruse, W., Munk, M., and Reitboeck, H. J., Coherent oscillations: a mechanism of feature linking in the visual cortex? Multiple electrode and correlation analyses in the cat, Biol. Cybern.,60:121-130, 1988.

[11] Eckmann, J. P. and Ruelle, D., Ergodic theory of chaos and strange attractors, Rev. Mod. Phys., 57:617-656, 1985.

[12] Freeman, W. J., Societies of Brains - A Study in the Neuroscience of Love Hate. and Lawrence Erlbaum Associates, Inc., Hillsdale, 1995.

[13] Freeman, W. J., A proposed name for aperiodic brain activity: stochastic chaos, Neural Networks, 13:11-13, 2000.

[14] Fujii, H., Ito, H., Aihara, K., Ichinose, N. and Tsukada, M., Dynamical cell assembly hypothesis - theoretical possibility of spatio-temporal coding in the cortex, Neural Networks, 9:1303-1350, 1996.

[15] Galarreta, M. and Hestrin, S., A network of fast-spiking cells in the neocortex connected by electrical synapses, Nature, 402:72-75, 1999.

[16] Gibson, J. R, Beierlein, M. and Connors, B. W., Two networks of electrically coupled inhibitory neurons in neocortex, Nature, 402:75-79, 1999.

[17] Gray, C., Engel, A. K., Koenig, P. and Singer, W., Synchronization of oscillatory neuronal responses in cat striate cortex: Temporal properties, Visual Neurosci., 8:337-347, 1992.

[18] Gray, C. M., Koenig, P., Engel, A. K., and Singer, W., Oscillator responses in cat visual cortex exhibit inter-columnar synchronization which reflects global stimulus properties, Science, 338:334-337, 1989.

[19] Han, S. K., Kurrer, C. and Kuramoto, Y., Dephasing and bursting in coupled neural oscillators, Phys. Rev. Lett., 75:3190-3193, 1995.

[20] Han, S. K., Kurrer, C. and Kuramoto, Y., Diffusive interaction leading to dephasing of coupled neural oscillators, Int. J. Bifurcat. Chaos, 7:869-876, 1997.

[21] Hindmarsh, J. L. and Rose, R. M., A model of neuronal bursting using three coupled first order differential equations, P. Roy. Soc. Lond. B. Bio., 221:87-102, 1984.

[22] Hodgkin, A. L., The local electric changes associated with repetitive action in a nonmedullated axon, J. Physiol., 107:165-181, 1948.

[23] Hodgkin, A. L. and Huxley, A. F., A quantitative description of membrane current and its application to conduction and excitation in nerve, J. Physiol., 117:500-544, 1952.

[24] Ikeda, K., Otsuka, K. and Matsumoto, K., Maxwell-Bloch turbulence, Prog. Theor. Phys. Supp., 99:295-324, 1989.

[25] Izhikevich, E. M., Neural excitability, spiking and bursting, Int. J. Bifurcat. Chaos, 10:1171-1266, 2000.

[26] Kaneko, K., Clustering, coding, switching, hierarchical ordering, and control in network of chaotic elements. Physica D, 41:137-172, 1990.

[27] Kaneko, K., Dominance of Milnor attractors and noise-induced selection in a multiattractor system, Phys. Rev. Lett., 78:2736-2739, 1997.

[28] Kaneko, K. and Tsuda, I., Complex Systems: Chaos and Beyond - A constructive approach with applications in life sciences, (Springer-Verlag, 2001).

[29] Kaneko, K. and Tsuda, I. eds. Chaotic Itinerancy, Focus issue in Chaos 13, 2003. 
[30] Kay, L., Shimoide, K., and Freeman, W. J., Comparison of EEG time series from rat olfactory system with model composed of nonlinear coupled oscillators, Int. J. Bifurcat.Chaos, 5:849-858, 1995.

[31] Lampl, I., Reichova, I., and Ferster, D., Synchronous membrane potential fluctuations in neurons of the cat visual cortex, Neuron, 22:361-374, 1999.

[32] Liljenstrom, H., Arhem, P. and Blomberg, C. eds., Workshop on "The Role and Control of Random Events in Biological Systems", Int. J. Neural. Syst., 7, 1996.

[33] Matsumoto, K. and Tsuda, I., Noise-induced order. J. Stat. Phys., 31:87-106, 1983.

[34] Milnor, J., On the concept of attractor, Commun. Math. Phys., 99:177-195, 1985.

[35] Morris, C. and Lecar, H., Voltage oscillations in the barnacle giant muscle fiber, Biophys. J., 35:193-213, 1981.

[36] Oseledec, V. I., A multiplicative ergodic theorem. Liapunov characteristic numbers for dynamical systems, Trans. Moscow Math. Soc., 19:197-221, 1968.

[37] Raffone, A. and van Leeuwen, C., Dynamic synchronization and chaos in an associative neural network with multiple active memories, Chaos, 13:1090-1104, 2003.

[38] Rinzel., J., Excitation dynamics: insights from simplified membrane models, Fed. Proc., 44:2944-2946, 1985.

[39] Rinzel, J. and Ermentrout, G. B., Analysis of neural excitability and oscillation. In Method in Neuronal Modeling: from Synapses to Networks, C. Koch and I. Segev, Eds., Cambridge, MA, MIT Press. 135-169, 1989.

[40] Roessler, O. E., Chaotic oscillations: An example of hyperchaos, in: Nonlinear Oscillations in Biology, ed. F. C. Hoppensteadt (Lectures in Appl. Math.) 141-156.

[41] Roessler, O. E., Knudsen, C., Hudson, J. L., and Tsuda, I., Nowhere-differentiable attractors, Int. J. Intell. Syst., 10:15-23, 1995.

[42] Rose, R. M. and Hindmarsh, J. L., The assembly of ionic currents in a thalamic neuron I. The three-dimensional model. P. Roy. Soc. Lond. B. Bio., 237:267-288, 1989.

[43] Rosen, R.,Life Itself, Columbia University Press, New York, 1991.

[44] Sano, M. and Sawada, Y., Measurement of the Lyapunov spectrum from a chaotic time series, Phys. Rev. Lett., 55:1082-1085, 1985.

[45] Sauer, T., Abstracts for SIAM Pacific Rim Dynamical Systems Conference, August 9-13, 2000, Hawaii, Maui, 51.

[46] Sauer, T., Chaotic itinerancy based on attractors of one-dimensional maps, Chaos, 13:947-952, 2003.

[47] Schweighofer, N., Doya, K. and Kawato, M., Electrophysiological properties of inferior olive neurons: A compartment model, J. Neurophysiol., 82:804-817, 1999.

[48] Skarda, C. and Freeman, W. J., How brains make chaos in order to make sense of the world, Behav. Brain Sci., 10:161-195, 1987.

[49] Sloper, J. J., Gap junctions between dendrites in the primate neocortex, Brain Res., 44:641-646, 1972.

[50] Tamas, G., Buhl, E. H., Loerincz, A. and Somogyi, P., Proximally targeted GABAergic synapses and gap junctions synchronize cortical interneurons, Neuroscience, 3:366-371, 2000.

[51] Tsuda, I., Koerner, E. and Shimizu, H., Memory dynamics in asynchronous neural networks, Prog. Theor. Phys., 78:51-71, 1987.

[52] Tsuda, I., Chaotic itinerancy as a dynamical basis of hermeneutics of brain and mind. 


\section{Tsuda et al.}

World Future, 32:167-185, 1991.

[53] Tsuda, I., Toward an interpretation of dynamic neural activity in terms of chaotic dynamical systems, Behav. Brain Sci., 24:793-847, 2001.

[54] Tsuda, I. and Umemura, T., Chaotic itinerancy generated by coupling of Milnor attractors, Chaos, 13:926-936, 2003.

[55] von der Marlsburg, C., The correlation theory of brain function, Internal Report 81-2, Goettingen, Max Planck Institute for Biophys. Chem., 1981.

[56] Wilson, H. R., Simplified dynamics of human and mammalian neocortical neurons, $J$. Theor. Biol., 200:375-388, 1999.

[57] Wilson, H. R., Spikes, Decisions and Actions, Oxford University Press, 1999. 